\title{
MONOLITHIC ELECTRONICS \\ FOR NUCLEAR AND HIGH-ENERGY PHYSICS EXPERIMENTS
}

\author{
Glenn R. Young \\ Oak Ridge National Laboratory, ${ }^{*}$ Oak Ridge, Tennessee 37831, USA
}

\section{- Invited Paper -}

5th Conference on the Intersections of Particle and Nuclear Physics

St. Petersburg, Florida

May 31 - June 6, 1994

\section{DISCLAIMER}

This report was prepared as an account of work sponsored by an agency of the United States Government. Neither the United States Government nor any agency thereof, nor any of their employees, makes any warranty, express or implied, or assumes any legal liability or responsibility for the accuracy, completeness, or usefulness of any information, apparatus, product, or process disclosed, or represents that its use would not infringe privately owned rights. Reference herein to any specific commercial product, process, or service by trade name, trademark, manufacturer, or otherwise does not necessarily constitute or imply its endorsement, recommanufacturer, or otherwise does not necessarily constitute or imply agency thereof. The views and opinions of authors expressed herein do not necessarily state or reflect those of the United States Government or any agency thereof.

"The submitted manuscript has been authored by a contractor of the U.S. Government under contract DE-AC05-84OR21400. Accordingly, the U.S. Government retains a nonexclusive, royalty-free license to publish or reproduce the published form of this contribution, or allow others to do so, for U.S. Government purposes."

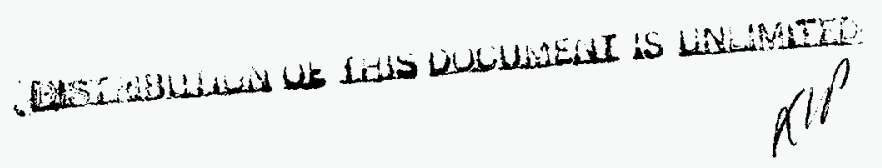

* Research sponsored by the U.S. Department of Energy under contract DE-AC05-84OR21400 with Martin Marietta Energy Systems, Inc. 


\section{DISCLAIMER}

Portions of this document may be illegible in electronic image products. Images are produced from the best available original document. 


\title{
MONOLITHIC ELECTRONICS \\ FOR NUCLEAR AND HIGH-ENERGY PHYSICS EXPERIMENTS
}

\author{
Glenn R. Young \\ Oak Ridge National Laboratory, \\ Oak Ridge, Tennessee 37831-6372
}

\begin{abstract}
Electronic instrumentation for large fixed-target and collider experiments is rapidly moving to the use of highly integrated electronics wherever it is cost effective. This trend is aided by the development of circuit building blocks useful for nuclear and high-energy physics instrumentation and has accelerated recently with the development of monolithic silicon chips with multiple functions on one substrate. Examples of recent developments are given, together with remarks on the rationale for use of monolithic electronics and economic considerations.
\end{abstract}

\section{INTRODUCTION}

A major consideration in the design of large experiments is the functionality and cost of the readout electronics required for the detector under consideration. Achieving large solid-angle coverage while retaining adequate granularity and detector thickness can often result in channel counts in excess of 100,000 and even 1 million for detectors of the scale required for SSC and LHC. Collider experiments have to ensure good reliability and significant self-diagnostic and calibration capabilities because of their scale, general inaccessibility for test and repair, and long operation periods; such capabilities obviously benefit fixed-target experiments also. The press to handle ever-higher luminosities to study high-mass, high- $p_{T}$ and/or rare processes has led to the development of fully pipelined architectures, which are extremely demanding in component count due to the deep buffers and extensive control logic required by them.

These considerations have spurred the development of multistage monolithic electronics, both in bipolar and CMOS processes, for use in present-day experiments. These circuits offer several advantages, including:

1. Reduced space requirements - this is particularly relevant both for silicon microstrip detectors and for hermetic fine-grained calorimeters.

2. Reduced power consumption - 1 million channels operating at 1 watt/channel (typical for CAMAC or FASTBUS) for a half year costs $\$ 0.24 \mathrm{M}$ at $\$ 60 / \mathrm{MWh}$ and results in a severe cooling problem; CMOS electronics of considerable complexity can achieve power dissipations less than one-tenth this amount.

3. Complexity - circuits with as many as a dozen gain ranges are possible, as are circuits with shaping, discrimination, timing, calibration, and LVL-1 trigger all incorporated onto one substrate. The "wiring" is also built into the chip, improving reliability. 
4. Elimination of delay cables - typical LVL-1 trigger formation delays are $500 \mathrm{~ns}$, corresponding to $100 \mathrm{~m}$ of RG-58 coaxial cable, resulting in one mega-meter of delay cable even in a modest calorimeter of $10 \mathrm{~K}$ channels; this can be replaced by monolithic analog storage units.

5. Cost - less than $\$ 50 /$ channel is possible for a system which includes preamp, shaper, discriminator, TAC, ADC, output buffer, calibration, and trigger formation; this must, however, be tempered with the observation that the development costs of monolithic electronics tends to restrict their deployment to detectors with more than $10 \mathrm{~K}$ channels.

The development of monolithic electronics in the nuclear and high-energy physics communities stretches back well over ten years, as evidenced, for example, by the early efforts for detectors such as the PEP-4 TPC at PEP and the SLD detector at the SLC. Considerable impetus came from groups developing the necessarily miniature electronics to read out silicon microstrip detectors at FNAL and CERN, followed by the large efforts for the LEP, HERA, TRISTAN, and Tevatron detectors. The field expanded considerably as a result of the R\&D efforts for SSC, RHIC, and now the LHC, with their emphasis on large channel counts, pipelined architectures, and extensive functionality. Examples are given in Table I below.

Table I. Examples of monolithic electronics developed for NP/HEP experiments

Analog Sampling/Delay Lines

LVL-1 trigger delay

DESY, Nevis, LBL, ORNL

Sampling for TPCs

LBL

Silicon-Strip Detectors

Early efforts: e.g., CDF vertex

CERN, FNAL, LBL, ORNL

Present: preamp/discr/trigger/

CERN, FNAL, LBL, ORNL

AMU/ADC

Time-Projection Chambers

Sampling for TPCs

Preamp/shaper/FADC for TEC/TRD BNL

Calorimetry

Preamp/shapers

Integrators

AMU

TAC/ADC

BNL, FNAL, CERN

ORNL, CERN

LBL, Nevis, DESY, ORNL, CERN

Nevis, LBL, ORNL, CERN 


\section{Drift/Straw Chambers}

Fast preamp/shaper/discr

Multihit TDC

MWPCs

Preamp/shaper/discr/latch

Pad Chambers

Preamp, rad-hard devices

\section{Speciality}

CFD/ LE discriminators

Trigger summing

Maskable calibration

Superconducting (!)

Josephson junction ( $\mathrm{Nb}, \mathrm{Nb}$-nitride)

tunneling devices

$100 \mathrm{GHz}$ counters, (F)ADCs, etc.
U. Penn

KEK, LeCroy, CERN, U. Paris

LeCroy/Yale

BNL, UT/ORNL

LeCroy, CERN, ORNL

many

many

Hypres Corp., Elmsford, NY

\section{EXAMPLES OF RECENT CIRCUITS}

\section{ASD-8 Chip (University of Pennsylvania)}

This is a combined preamplifier, shaper, and discriminator eight-channel chip developed for drift chambers. The circuit is implemented in a bipolar process in order to achieve the very fast rise and fall times needed for precise tracking and good two-track separation using drift chambers and straw tubes. The initial development was aimed at the strawtube tracker of the SDC experiment at SSC. A block diagram [1] is shown in Fig. 1. The design goals of the chip include:

0.75-ns time resolution (which translates to 50-100 microns space-point resolution, depending on chamber gas)

Double pulse resolution of $20 \mathrm{~ns}$ (i.e., 1- to 2-mm spatial separation)

Semigaussian shaping to minimize baseline shifts

Pole-zero and ion-drift tail cancellation

Threshold programmable from 1-10fC

Programmable disable mask

Power dissipation of less than $10 \mathrm{~mW} /$ channel

Radiation resistance to better than $1 \mathrm{MRad}$ and $10^{14} \mathrm{n} / \mathrm{cm}^{2}$ 


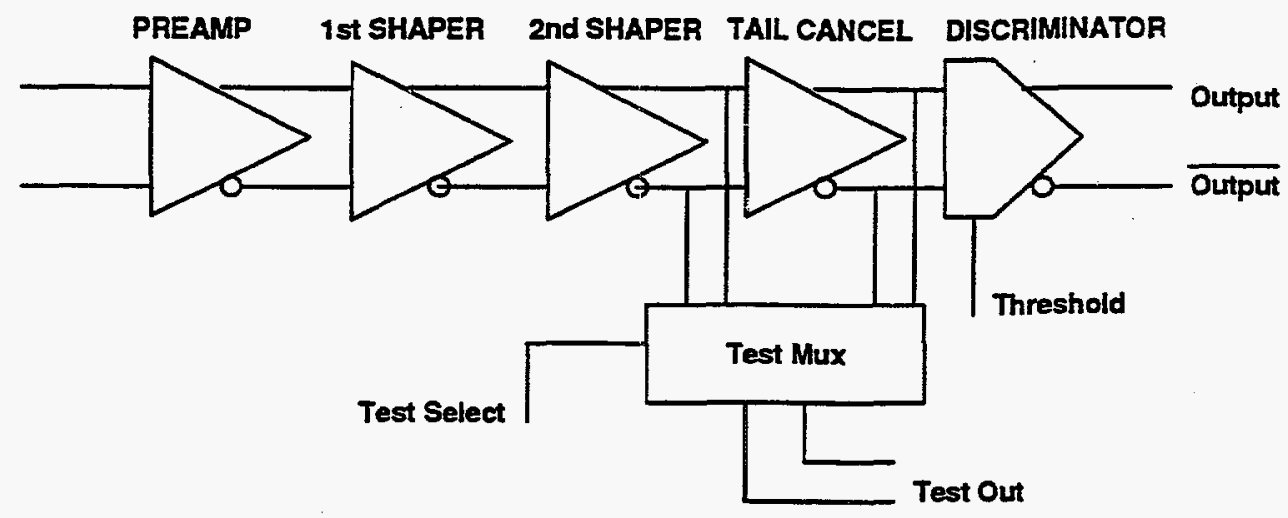

Figure 1: Block diagram of the ASD-8 circuit.

These goals are met. Cross-talk of order only $1 \%$ even at $1.5 \mathrm{fC}$ threshold has been observed. A sample pulse shape observed from an early version is shown in Fig. 2.

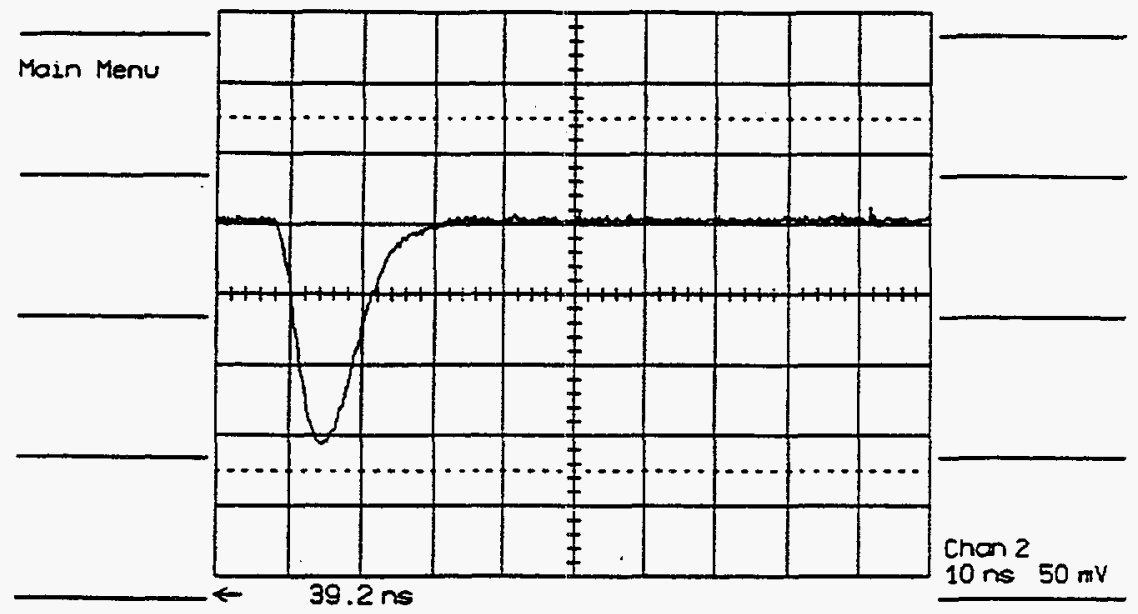

Figure 2: ASD-8 second shaper response for a 1fC impulse input.

\section{TMC-Series Pipelined TDCs}

A series of pipelined TDCs has been developed at KEK, again with initial impetus from the SDC-tracking effort. A diagram [2] of the architecture of these circuits is shown in Fig. 3. The concept underlying this design is to use a high-speed shift register technique to determine when a discriminator signal occurs with an accuracy of better than $1 \mathrm{~ns}$. Instead of a $1-\mathrm{GHz}$ clock (which would require use of GaAs technology), actual implementation in a CMOS process uses a series of rows of memory cells. Successive (logical) rows of 32 cells each are selected by a $30-$ to $40-\mathrm{MHz}$ clock, and within the 
period of this clock, the phase of the detector signal is determined by comparing it to a copy of the clock signal sent down a delay line with taps at 1-ns intervals. The pattern of fired cells thus records the times of the leading and trailing edges of the discriminator signal from the detector. This pattern is stored in a dual port memory some 128 cells deep to allow for LVL-1 trigger delay of up to $4 \mu \mathrm{s}$. Patterns selected by a valid trigger for readout are encoded to conserve on the number of output pins on the 4-channel chip. Both leading and trailing edges are reported, together with a tag bit to speed up later sparse-data scans and zero-suppression operations.

(a) High-Speed Shitt Registers

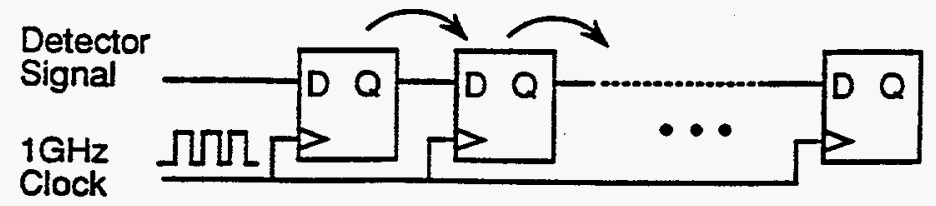

(b) TMC

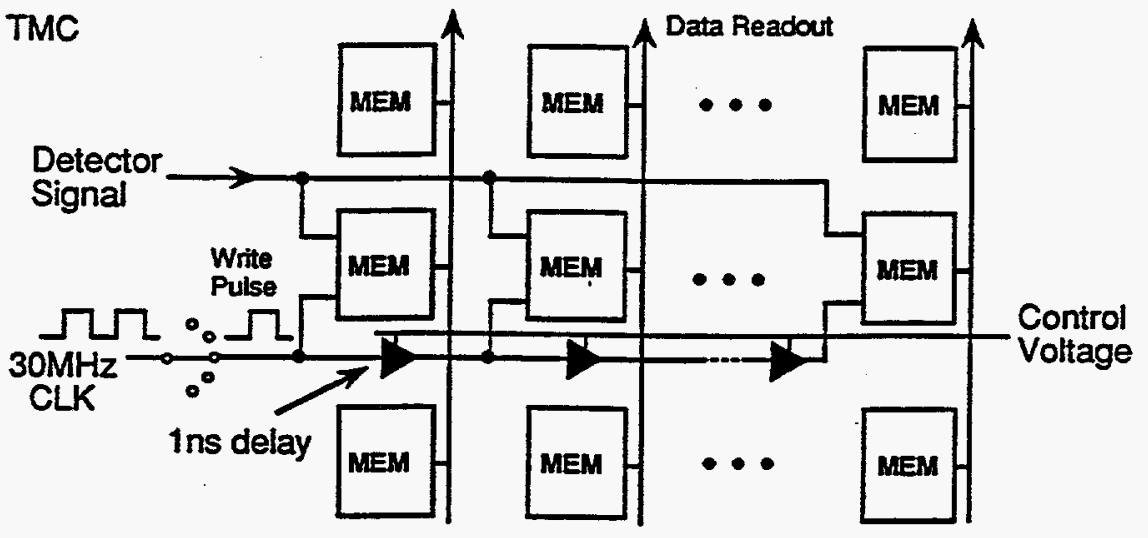

Figure 3: Architecture of TMC-series of pipelined TDCs: (a) high-speed shift register; (b) time memory cell.

The chip incorporates a phase-locked loop and feedback to ensure long-term stability of the time base relative to an external global reference clock signal. This also permits a variation of the operating frequency from $30-40 \mathrm{MHz}$, which allows the same chip to be used for a range of beam-crossing frequencies as would be encountered at, e.g., different colliders. The latest versions of this chip achieve better than $0.3-n s$ resolution and provide LVL-1 trigger delays in excess of $4 \mu \mathrm{s}$.

\section{Broad-Range Integrator for Calorimetry}

A standard problem in calorimetry at colliders is obtaining a system with 8-9 bits precision at any given point in an overall dynamic range of 18-22 bits. A novel technique for dealing with this in the case of calorimeters read out by photomultipliers has been developed at FNAL for the scintillating-tile calorimeter proposed for SDC. This technique takes advantage of the fact that photomultipliers are good current sources 
over a large dynamic range and implements a current-sharing technique to achieve 10 bits of pre-ranging in a pipelined fashion. A block diagram is shown in Fig. 4 (Ref. [3]).

The anode signal from a PMT is fed into a binary-weighted, stabilized and balanced, current splitter which covers ten binary steps. The split currents are integrated on a bank of capacitors, whose voltages are then compared in parallel to a set of ten reference voltages. The resulting pattern is then encoded to form a four-bit exponent of the ultimate ADC output and simultaneously drives an analog multiplexer. The multiplexer selects the appropriate stored charge to be digitized by a FADC of 8 to 12 bits to provide the mantissa of the ADC output.

Actual implementation required use of a four-stage pipelined scheme in order to allow for settling times for the various logical steps shown in Fig. 4. The clock for the pipeline proceeds at the full speed of the SSC (or LHC) beam-crossing clock, meaning that (assuming a sufficiently fast FADC is employed) fully-digitized data from the calorimeter can be prepared with as many as 12 bits precision over a 22-bit range at the full beamcrossing rate. The utility of such a scheme in forming fast, accurate, pipelined triggers to look for jets and high- $p_{T}$ particles in a collider or fixed-target environment is clear.

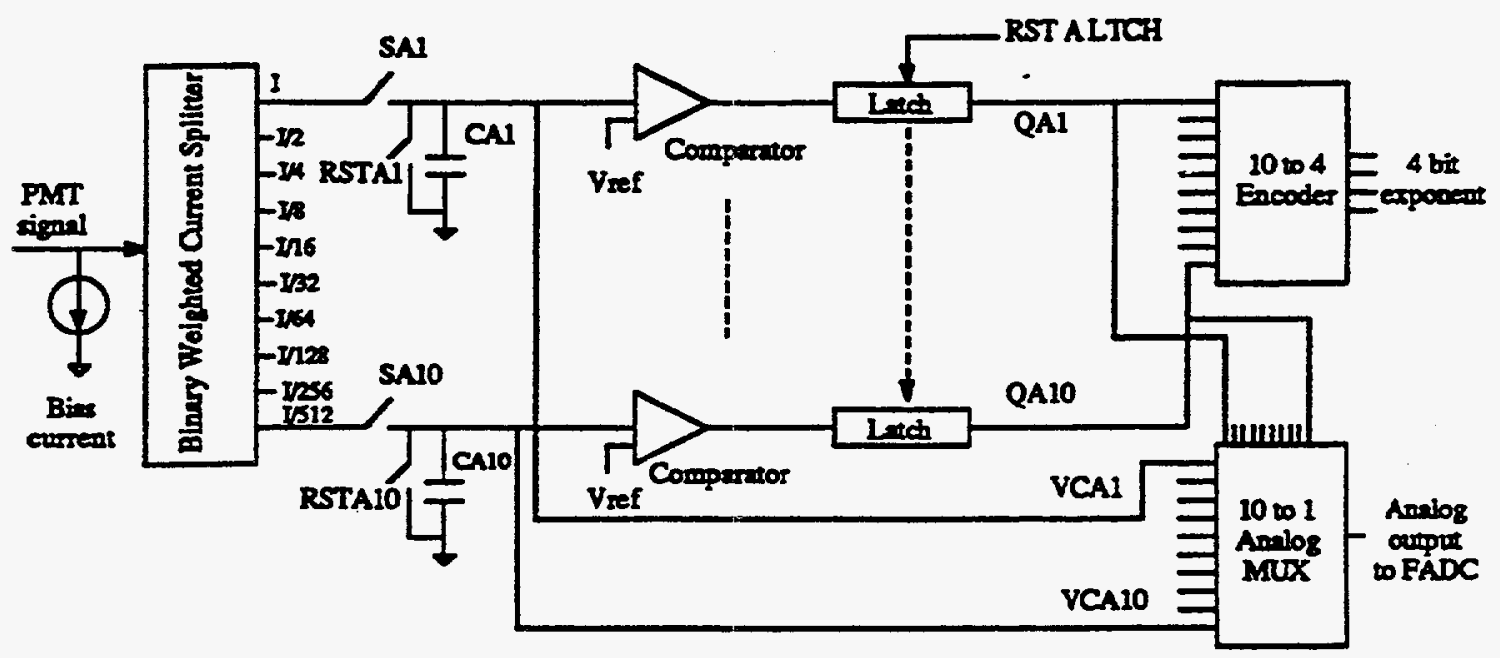

Figure 4: Simplified block diagram of broad-range integrator.

Electronics for a Lead-Glass Calorimeter

A pair of chips have been developed to read out a $10 \mathrm{~K}$-element lead-glass electromagnetic calorimeter used as the photon detector in CERN experiment WA98. Each glass block, or "module," is read out by means of its own photomultiplier tube. The anode pulses from these photomultipliers are observed to have a nearly Gaussian shape, with 10 -ns rise and 10 -ns fall ( $10 \%$ to $90 \%$ amplitude), and a small-amplitude tail extending to nearly $300 \mathrm{~ns}$ after the event. The measured pulse-height resolution behavior of a $3 \times 3$ group of modules can be described as $\frac{\sigma_{E}}{E}=\frac{6 \%}{\sqrt{E(\mathrm{GeV})}}+1 \%$ as measured with electrons from 0.1 to $70 \mathrm{GeV}$. The timing resolution of a module has been measured to be $\delta_{t}(\mathrm{rms}) \sim 280$ ps at $2-3 \mathrm{GeV}$ for electrons. 
The electronics were designed to provide the following: (1) the $300-400 \mathrm{~ns}$ of LVL-1 trigger delay required in WA98, without the use of delay cable, (2) digitization of the integrated anode charge with 10 bits precision over a 13-bit range, (3) timing information with 100-ps electronics resolution or better, (4) maskable calibration pulsers for the amplitude and timing channels, and (5) cluster-trigger with adjustable energy threshold. The custom part of the implementation consisted of two ASICS: a "Preamp" chip and an AMU/TAC/ADC ("ATA") chip, described below.

The principle of the pulse height measurements is to sample at a fixed frequency the output of a circuit which integrates the anode current as a function of time, and then to store those samples in a switched-capacitor analog memory unit (AMU) pending receipt of a positive trigger decision from external logic in the experiment. Receipt of a valid trigger causes a sample recorded before the event (the "pre-" sample) and one recorded following the event (the "post-" sample) to be selected in succession from the $A M U$, digitized by the $A D C$, and the results sent to the data acquisition system. The integrator output is sampled, and thus the AMU is written, at $20 \mathrm{MHz}$ in the present system. The AMU is 16 cells deep, implying that the trigger decision must be received no more than $750 \mathrm{~ns}$ after the event.

The timing channel was designed with a constant-fraction discriminator in order to provide walk of $\leq 300$ ps over a dynamic range in pulse height of 100:1. This pulseheight range is set to correspond to a photon energy range of $50 \mathrm{MeV}$ to $5 \mathrm{GeV}$ in actual operation. The TAC is operated in common-start mode, in order to avoid having channels rendered dead because of an uninteresting pulse occurring since the previous accepted event but prior to the next accepted event. This required development of a delay, stable to 1 part in $10^{3}$, for the discriminator output which could provide the 300 to 400 -ns delay required for LVL-1 trigger formation.

The module cluster trigger circuits are designed to form first a disjoint set of $2 \times 2$ sums of module pulse heights, i.e., produce a single nonoverlapping tiling of the $10 \mathrm{~K}$ modules. These $2 \times 2$ sums are then combined into a set of overlapping $4 \times 4$ sums in order to provide full trigger coverage for photons even if they should land near edges of modules or of the $2 \times 2$ regions themselves. This trigger is implemented in a currentsumming mode and includes a programmable threshold controlled by a 6 -bit DAC, all included on the "Preamp" chip. Board-level traces and board-to-board jumpers are included to form trigger sums which extend beyond the confines of a single ASIC or even a given electronics board.

The Preamp ASIC includes eight channels of charge-integrating amplifiers followed by $\mathrm{x} 1$ and $\mathrm{x} 16$ amplifiers which in turn drive the two AMU inputs and thus provide the dual-ranging $\mathrm{ADC}$ behavior desired. There are also eight timing filter amplifiers each followed by a constant fraction discriminator. Each channel has a maskable, triggerable calibration pulser set by a 6-bit on-chip DAC and is also connected to a multiplexer used for observation of the input anode signal. A block diagram of the Preamp ASIC is shown in Fig. 5.

The constant fraction discriminator uses on-chip, lumped-element shaping to remove the need for external delay cables. Auto-zeroing is included to eliminate any need for walk adjustment. The circuit is optimized for the 10-ns rise/fall times of the FEU-84 PMTs used, so these features remove the need for any adjustment other than setting a firing threshold, which is done using one of the six-bit DACs included on the Preamp 
chip. The walk is observed to be \pm 300 ps over the entire $100: 1$ dynamic range of $-30 \mathrm{mV}$ to $-3 \mathrm{~V}$ for which the discriminator is designed. Over limited dynamic ranges of $6: 1$, walk less than $100 \mathrm{ps}$ has been observed.

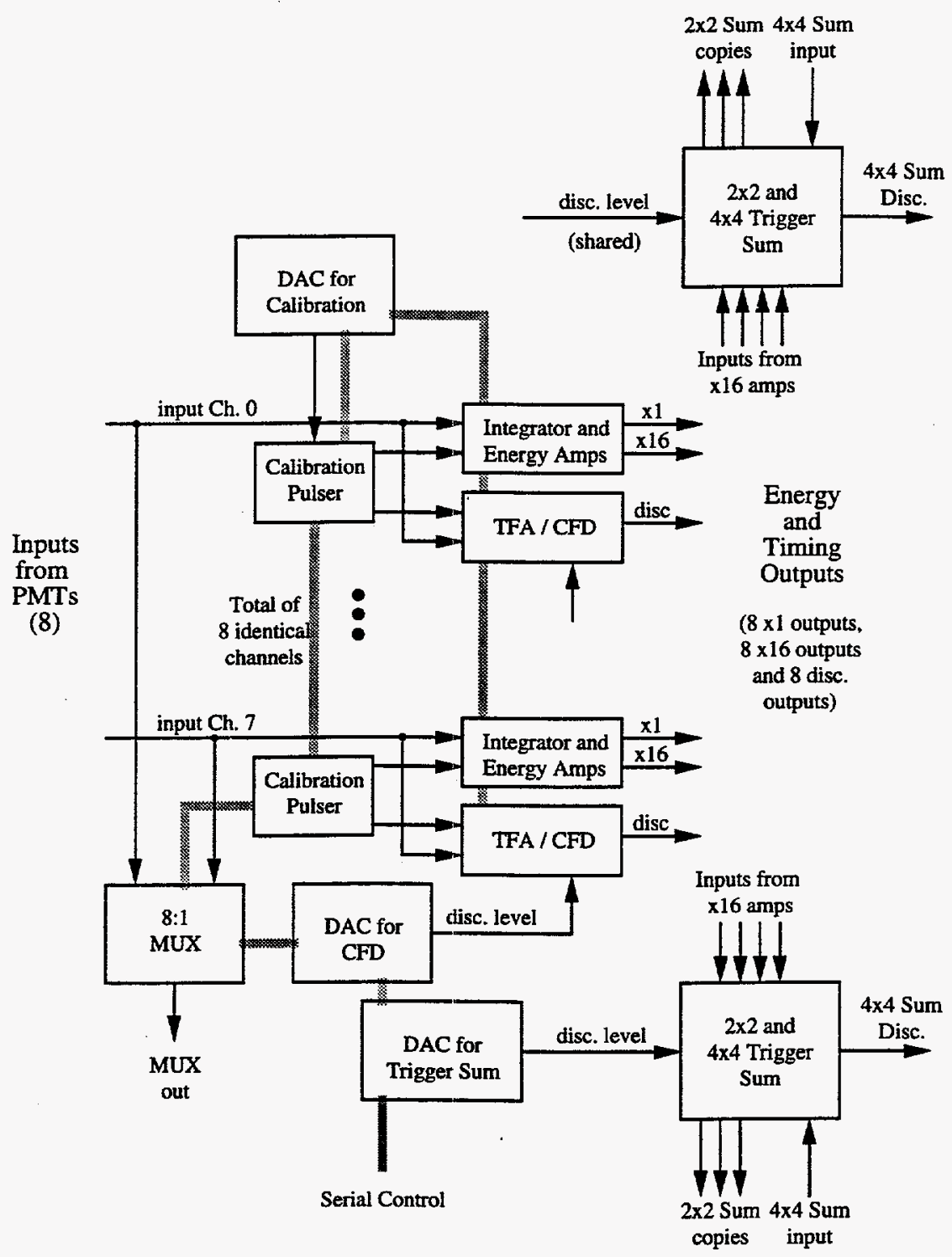

Figure 5: Block diagram of lead-glass Preamp chip.

The AMU is a voltage-write, voltage-read type. It operates at $20 \mathrm{MHz}$ (writing) and is capable of a 25-ns write to a given cell. The typical memory "gain" of the AMU is 0.956 . Cell-to-cell pedestal variation of $3.8-\mathrm{mV} \mathrm{rms}$ has been measured for a single pipeline of the prototype AMU, using addressing driven by an FPGA. This corresponds to $0.8 \mathrm{LSB}$ for 10 -bit operation. The measured integral nonlinearity is $\pm 0.05 \%$.

The TAC range is adjustable from $50 \mathrm{~ns}$ to $1000 \mathrm{~ns}$ using an external control voltage. The noise has only been measured to date using a 10-bit ADC; all events (for in excess of 10,000 counts) fell into one $\mathrm{ADC}$ channel for this measurement, indicating that at 
the \pm 2 sigma level, the rms noise is of order 25 ps or less. The TAC resets to better than 1 LSB (out of 10-bits) in 50 ns.

The ADC is a multichannel Wilkinson-type architecture with 10-bit counters. The ramp generator and clock are shared among the 24 channels. The clock is operated at $100 \mathrm{MHz}$ nominally, but has been tested successfully up to $170 \mathrm{MHz}$. The integral nonlinearity over the full range is $0.2 \%$, and the measured differential nonlinearity is $33 \%$, for an rms of 0.6 channels. The design allows a straightforward extension to 12 bits, faster clocks, and more channels.

\section{COSTING CONSIDERATIONS}

Development of such circuits is not for the faint of heart. A new design can require 2-3 man-years of effort, and a modification can require 2-6 man-months to complete. Better than half the effort is commonly invested in testing the design to see if it meets specifications; the capability to do this has to be built in to the design, as probing all points inside the chip is not possible. An investment in the necessary computer-aided design tools is mandatory; fortunately, public-domain tool sets exist.

Prototyping costs depend heavily on the process used. Bipolar and radiation-hard processes typically require the user to shoulder the full cost of a run, which can approach $\$ 100 \mathrm{~K}$. CMOS designs which are not rad-hard can be prototyped using the MOSIS facility sponsored by DARPA, or may be handled by silicon foundries which offer such a service. The MOSIS facility is designed to encourage student participation from universities and can cost as little as a few hundred dollars for four prototype $4 \mathrm{~mm}^{2}$ chips. A disadvantage lies in slow turn-around, but it does provide access to several foundries. Private commercial arrangements can cost between $\$ 3$ and $\$ 15 \mathrm{~K}$ for prototypes from 4 $\mathrm{mm}^{2}$ to $64 \mathrm{~mm}^{2}$ in size but offer reliable turn-around time.

Production costs at a silicon foundry for large runs typically have nonrecurring costs of $\$ 30-45 \mathrm{~K}$ for masks, $\$ 20-30 \mathrm{~K}$ for probing, and $\$ 30 \mathrm{~K}$ for a minimum set of ten 4 -inchdiameter wafers. A given wafer produces anywhere from 50-400 raw die, depending on die size. However, once a project's requirements result in a need for more than ten wafers, economies of scale set in very rapidly: extra wafers cost typically $\$ 1 \mathrm{~K}$ each, and the number ordered can increase without limit as long as the foundry's production can keep up. Adding packaging and testing of the final product (which is mandatory, as yields for $16-\mathrm{mm}^{2}$ die, for example, are typically only $50 \%$ ), the marginal cost of a die can fall to well under $\$ 50$, to as low as $\$ 2$ each (packaged) for small die. This then can lead to per channel marginal costs of $\$ 1-\$ 10$, which is extremely attractive, more so when the functionality per chip described above is considered.

\section{ACKNOWLEDGEMENTS}

I have benefitted from numerous discussions from the actual practitioners in this field, including C.-Y. Chi and W. Sippach of Nevis Laboratory, Y. Arai of KEK, M. Newcomer of University of Pennsylvania, P. O'Connor and J. Harder of BNL, and especially my colleagues in the Instrumentation and Controls Division at ORNL for the WA98 leadglass project, G. T. Alley, C. L. Britton, L. G. Clonts, M. S. Emery, M. N. Ericson, M. L. Simpson, J. W. Walker and A. L. Wintenberg. 
* Research sponsored by the U.S. Department of Energy under contract DE-AC05$840 R 21400$ with Martin Marietta Energy Systems, Inc.

\section{REFERENCES}

1. E. Gerds, F. M. Newcomer, R. Van Berg, J. Van der Spiegel, and H. H. Williams, Proc. Int. Conf. on Electronics for Future Colliders, May 19-21, 1992, LeCroy Corp., eds. George J. Blanar and Richard A. Summer, p. 105.

2. Y. Arai, F. Sudo, and T. Emura, Proc. Int. Conf. on Electronics for Future Colliders, May 4-5, 1993, LeCroy Corp., eds. George J. Blanar and Richard A. Summer, p. 33.

3. R. J. Yarema, W. Foster, J. Hoff, M. Sarraj, and T. Zimmerman, Proc. Int. Conf. on Electronics for Future Colliders, May 19-21, 1992, LeCroy Corp., eds. George J. Blanar and Richard A. Summer, p. 131. 